\title{
Lumen
}

Selected Proceedings from the Canadian Society for Eighteenth-Century Studies

\section{Pierre Potier à Windsor, une nouvelle frontière de la culture française au dix-huitième siècle}

\section{Robert Toupin}

Volume 16, 1997

Freedom and Boundaries

Émancipation et frontières

URI : https://id.erudit.org/iderudit/1012441ar

DOI : https://doi.org/10.7202/1012441ar

Aller au sommaire du numéro

Éditeur(s)

Canadian Society for Eighteenth-Century Studies / Société canadienne d'étude du dix-huitième siècle

ISSN

1209-3696 (imprimé)

1927-8284 (numérique)

Découvrir la revue

Citer cet article

Toupin, R. (1997). Pierre Potier à Windsor, une nouvelle frontière de la culture française au dix-huitième siècle. Lumen, 16, 83-92.

https://doi.org/10.7202/1012441ar 


\section{Pierre Potier à Windsor: une nouvelle frontière de la culture française au dix-huitième siècle}

Si vous aviez quitté La Rochelle sur le vaisseau du roi le Rubis, au printemps de l'année 1743, vous seriez arrivé à Québec après 108 jours de traversée - un trajet que l'on parcourt en six ou sept heures aujourd'hui. Quitter son village; sa famille; son univers familier - pour quelle aventure? et combien d'inconnues? Certes! il y eut un appel; une vocation; un risque redoutable! Et pourquoi pas?

Le jésuite belge Pierre Potier, missionnaire chez les Hurons et Français de la région de Windsor de 1744 à 1781, peut être considéré, au territoire frontière du Détroit, comme le prototype d'une aventure à la fois missionnaire et intellectuelle d'exception, comme le révèlent ses écrits personnels. ${ }^{1}$ Une oeuvre écrite considérable, comprenant environ 20,000 pages, d'une écriture serrée, d'où se détachent assez nettement les composantes d'une activité pastorale et surtout littéraire étonnante. Les soixante-dix cahiers de notes du Père Potier recouvrent des domaines multiples: théologie (les traités conventionnels y figurent verbatim), philosophie, morale, Écriture Sainte, linguistique (latine, française, huronne) et, en particulier, le secteur des sciences. En outre, il y a un important dossier portant sur l'administration de la mission (livres de comptes, registres de catholicité des Hurons et Français, correspondance, bibliothèque). Un vaste ensemble de recueils, à la frontière du savoir encyclopédique.

Nous avons affaire ici à un témoin attentif du Nouveau Monde, tant sur le plan géographique que culturel. Potier a enregistré une toponymie qui traverse, depuis la Belgique et la France, le corridor de l'Atlantique Nord, le golfe Saint-Laurent, les routes vers l'ouest, en particulier la région du Détroit et nombre de routes vers le sud, aux États-Unis. Tout au long de la route, il y a quantité de noms français, de familles françaises et amérindiennes, dont Potier s'approprie la réalité, en la fixant sur papier vergé. Il y a là une série de chemins ou itinéraires, sur terre et sur mer, depuis l'ancien monde jusqu'au nouveau. 
Toute cette écriture, parallèle à l'activité d'un pasteur vigilant, se construit dans un poste isolé des grands lacs, autour du village des Hurons, voisin de l'établissement français (à Détroit et à Windsor). Le passionné d'écriture et de lecture articule le projet de notation comme un administrateur d'empire, retenu captif au rivage des choses de l'esprit: celui des lettres, des langues, des sciences, sans parler des ouvrages de spiritualité. Dans ce décor, on croirait que le temps de rédaction fait concurrence au temps réservé à la responsabilité du pasteur. D'un côté le souci de suivre attentivement le progrès de la mission, comme tout bon missionnaire se doit de le faire et, de l'autre, construire discrètement l'édifice du savoir, comme d'autres explorateurs et savants l'ont fait, jésuites compris, à l'instar des rédacteurs des Relations des Jésuites ou, encore, en suivant la trace des Lettres édifiantes et curieuses.

\section{Un homme de lettres gallo-belge}

Né à Blandain, Belgique, petit village voisin de la Picardie, Pierre Potier (1708-1781) était lui-même un homme de la frontière, puisque son village natal touchait à celle de la France. Ses études se poursuivront dans les institutions de la province gallo-belge de la Compagnie de Jésus.

Potier ne nous dit jamais qu'il est wallon. Le père Pierre-Daniel Richer, supérieur de la mission huronne de Lorette, l'appellait «bouche-belgique». Non seulement écrivait-il beaucoup, mais il parlait beaucoup. L'aumônier récollet du fort Pontchartrain, Bonaventure Léonard, l'invitait ainsi: "Vous viendrez chez moi faire marcher votre remuante langue». Trace précieuse d'une prise de parole, aussi remuante que la plume, toujours en instance d'activité créatrice, cimentée à la passion de l'écriture.

Parlons des années d'étude et de la formation du jeune homme. Dans les collèges jésuites de l'Ancien Régime, la formation était essentiellement classique, inspirée des modèles de la Renaissance et du modus parisiensis, répandus par les Jésuites dans les pays d'Europe et dans les territoires de missions, aux colonies. Le latin, langue de Cicéron et de Virgile, avait priorité sur le français, et tous les écoliers destinés aux fonctions importantes, tant dans l'État que dans l'Église, devaient assimiler cette forme de culture, considérée comme nécessaire à l'équilibre de l'esprit, à la formation du goût, du caractère et du jugement. Privilège réservé à une classe sociale très restreinte, en particulier celle de la magistrature et des milieux bourgeois.

Quant à ceux que la littérature classique ou française attirait, il se créait dans leur esprit des habitudes du côté de l'apprentissage des langues. Lorsque Potier se livre à la langue huronne, c'est la connaissance 
du latin qui intervient pour reconstituer la grammaire et le lexique de cette langue. Dans le sillage des experts de la langue huronne, tels que Jean de Brébeuf, Joseph-Marie Chaumonot et autres missionnaires en Nouvelle-France, Potier se donne la mission de continuer et d'enrichir le corpus de cette langue, sans doute fort difficile pour les Européens.

Voilà qui nous amène à ses choix personnels, i.e. à cette passion d'accumuler le savoir, à le constituer, en intégrant à cette formation classique un ensemble de connaissances tirées des lectures dans presque tous les domaines touchés par les ouvrages du temps. Les cahiers de notes de Potier sont sur ce point assez clairs et illustrent nettement le contenu de ses études et lectures.

Il y a deux territoires : (1) les études communes à tous les candidats à la Compagnie de Jésus: lettres classiques, philosophie, théologie, ouvrages de spiritualité et de pastorale, Écriture Sainte, morale; (2) Les études et lectures personnelles, qui touchent aux domaines suivants: linguistique latine, grecque, huronne et française. Peter Halford a édité le lexique Potier du parler français au dix-huitième siècle. Un vaste domaine littéraire occupe la plume de Potier. Il note les oeuvres des prosateurs et des poètes. Au fil de la lecture des journaux et gazettes du temps, les notes historiques abondent en chroniques du temps, suivent de près les affaires d'Europe, de la France en particulier. D'autre part, la documentation géographique vient enrichir la toponymie française de l'Amérique du Nord.

Le secteur des sciences est plus vaste encore, aborde l'univers complexe de la physique, terme générique désignant l'ensemble des sciences de la nature et comprenant : la mécanique, l'astronomie, la mathématique, la médecine, la chirurgie, la pharmacopée, l'apothicairerie, la botanique, la minéralogie, l'hydrographie, l'architecture, sans parler des parties multiformes d'un dictionnaire personnel, où s'amoncellent les pièces d'une terminologie souvent démultipliée, selon la variété des emplois de chaque terme. Trois langues dominent cette passion d'écrire: le français, le latin, le huron. Le tout condensé dans une écriture ultra fine (jusqu'à 40 lettres $\mathrm{au} \mathrm{cm}^{2}$ ).

\section{Une Amérique française au Détroit}

Depuis le départ de Belgique jusqu'au vaste territoire de l'empire français d'Amérique, Pierre Potier se fera un devoir de suivre de près les noms de lieu, les distances, de noter le temps requis entre les étapes, créant pour l'avenir un journal de bord des plus utiles pour cartographes et explorateurs. Ainsi, les voies maritimes, les routes de canot ou les routes par terre font partie des recueils Potier et demeurent une source importante de la riche toponymie française du dix-huitième siècle. 
Quel que soit le territoire visé, le guide du voyageur suit les chemins d'autres explorateurs et s'approprie les noms de lieu, dont un grand nombre se retrouvent aujourd'hui, noms quelquefois inspirés des langues amérindiennes. Pour s'en tenir à la partie qui concerne le sud de $l^{\prime}$ Ontario, voici, extraits de la rédaction Potier $^{2}$, quelques traces du parcours des lieux français:

a) Depuis les chutes Niagara: la Pointe aux Roches, la Pointe à Bino, le Pain de Sucre, la rivière au Bordel, la rivière à l'enclume (ou à la Chaînette), la Pointe aux Biches, le Chenail au Rigolet, la Longue Pointe, le Grand Bois, les Grands Écors, la rivière à la Voile, la rivière à la Barbue, la rivière à la Chaudière, la rivière à Madame Tonti, la Pointe au Fort, la Pointe à Desjardins, la rivière à la Chenaie, le Campement Vert, la Pointe aux Pins, la Pointe Pelée, la rivière aux Cèdres, la Presqu'Île, la Pointe de Sable, l'île aux Bois-Blancs.

b) Du Détroit au fort Saint-Joseph (Niles, Mich.): la petite Rivière Rouge, les Castors ou les Arbres Matachiés, les Abbatis du Diable, la rivière aux Hurons, la Saline (lieu du village des Poutéouatamis), le Lac des Mamelles, le Vermillon, le Lac aux Grés, la grande rivière Ouachtanon (aujourd'hui Wachtenaw).

c) Du Détroit au fort des Miamis (Fort Wayne): le lac Érié, la baie d'Onanguissé, la rivière des Miamis (Maumee River), la Roche Debout, le Grand Rapide, les Pays Plats, la Grande Glaise (Auglaise River et Defiance), le Marais de l'Orme (transformé plus tard en Marie de Larme, nom d'une rivière voisine du marais, sans doute une trouvaille $d^{\prime} u n$ pauvre artisan des massacres linguistiques), le Grand Rapide, la Glaise à Roye, la Roche Fendue, le Rapide du Boeuf, les Mamelles, le Grand Détour.

Les guides de voyage de Potier ajoutent une liste des établissements dépendant du Mississippi et une liste des pointes et rivières sur le territoire des Grands Lacs, avec traduction en langue huronne. Un souhait: que les spécialistes des langues amérindiennes procèdent à $l^{\prime}$ analyse linguistique de ces noms et revèlent les images miroitantes du parler amérindien.

\section{Une mission parmi les Hurons et Français}

C'est avant tout pour continuer l'oeuvre d'évangélisation que Pierre Potier, en 1744, se rend à la mission Notre-Dame de l'Assomption, située à l'Ile aux Bois-Blancs (Boblo-Island), voisine du Fort Pontchartrain, dans la rivière Détroit.

Depuis la fondation de Détroit par le sieur de Lamothe Cadillac en 1701, les Hurons-Pétuns du Détroit avaient occupé trois sites : le premier site à côté du Fort Pontchartrain (de 1701 à 1742), le suivant à l'Ile aux 
Bois-Blancs (de 1742 à 1747), le troisième à la Pointe de Montréal (à Sandwich, aujourd'hui Windsor).

Un document d'une valeur exceptionnelle, rarissime semble-t-il, c'est bien le double recensement de la nation huronne, pour l'année 1747. Il a été inséré parmi les documents en langue huronne (Écrits, doc. 13). Ce texte contenait les noms des occupants des 45 cabanes huronnes, tant à l'Île aux Bois-Blancs que dans les villages des Champs, situés au sud du lac Érié. La population huronne était répartie en deux villages: le Petit Village et le Grand Village. Les chefs de cabane sont souvent des huronnes, qui ont survécu à leur conjoint. D'ailleurs, dans le Conseil de la nation, divisée en trois bandes, se trouve un nombre presqu'égal d'hommes et de femmes.

Du côté français, nous avons ici, dans un territoire que la France occupe et agrandit tout au long de la première moitié du dix-huitième siècle, une présence particulièrement active des familles et des soldats français, tant du côté de Détroit (au fort Pontchartrain et dans la région) qu'à la Pointe de Montréal et dans son voisinage, au lieu dit La Petite Côte, du côté de Amherstburg.

Nous connaissons Hurons et Français grâce aux registres de la mission. ${ }^{3}$ Ces registres donnent les noms des baptisés, des parents, des parrains et marraines, tant des Hurons (1728-1781) que des Français (1762-1781). Le relevé des actes pour les mariages entre Français et Françaises signale le lieu d'origine des conjoints. Les signatures nous situent sur le plan de l'alphabétisation. En fait, c'est celle de la France à l'époque.

Sur le plan de la pastorale, notons que Potier, qui a hérité des listes de cantiques et prières de ses prédécesseurs, en a reconstruit tout le répertoire, notant de surcroit avec grand soin les prescriptions du Rituel de Québec. Il veille attentivement à la manière dont les Hurons s'appliqueront à faire un testament en faveur des parents défunts, occasion souvent renouvelée en faveur de ceux qui ont quitté ce monde. Afin d'assurer la recommandation de l'âme des proches disparus, leurs noms sont inscrits et ce, durant plusieurs années, expression tangible d'une douleur partagée, tradition particulièrement vive parmi les nations indiennes.

Disons qu'une fois intégrés au christianisme par le baptême, la fidélité de la nation tend à se consolider si le missionnaire est vigilant. En fait, les chefs, les anciens et anciennes se considèrent parfois comme les gardiens de la foi. Néanmoins tout peut se dégrader si la Robe Noire quitte les lieux. Malheureusement les documents gardent le silence sur le phénomène de la résistance à l'enseignement chrétien, sans doute contesté vivement dans les débuts d'une mission. Une question difficile à interpréter. 
Signalons que la journée du missionnaire comprend plusieurs heures de prière, de cérémonies, d'enseignement du catéchisme, de visite des malades et mourants. Comme Potier avait été directeur de chorale, on doit supposer qu'il veillait à la qualité du chant, qui comprenait musique grégorienne et chants traditionnels des églises de France ou de Belgique. Mais les documents n'indiquent presque jamais comment le message chrétien a été accepté, intégré. Il y avaient toujours les mêmes enseignements, les mêmes liturgies, les mêmes manières de procéder. Avec cette différence toutefois: on tenait compte du goût des amérindiens pour les cérémonies plus somptueuses, pour le chant, pour les images et la statuaire.

Bien que Potier a beau être un connaisseur de la langue huronne, il se contente d'en reconstruire la grammaire et le lexique, sans faire d'exposé spécial sur l'originalité et la beauté de cette langue. On y trouve toutefois davantage de signes diacritiques que dans les écrits des prédécesseurs, Brébeuf et Chaumonot. Il y a là un immense territoire d'enquête!

\section{Quelques sentiers du côté de la culture française}

Comme on peut le constater, le travail du père Potier était en somme divisé en deux parties : (1) l'activité pastorale parmi les Hurons et Français; (2) le temps réservé à la lecture et à l'écriture, une passion qui nous place sur le parcours de sa vie intellectuelle, celle d'un homme de lettres, voire même d'un encyclopédiste, sur le ton mineur, tissant une toile qui s'agrandit constamment. Le courrier arrivant au Détroit contient quelques gazettes de France, les Étrennes mignonnes, le Courrier $d u$ Mardi, etc., des ouvrages de science, d'histoire, de littérature, de spiritualité. Si les notes de lecture s'accumulent, on n'y trouve aucune tentative pour contester les affirmations des auteurs. Quel que soit le sujet abordé, le contenu nous revient, souvent textuellement, sans discussion ou interprétation, qu'il s'agisse des lettres, des sciences, de la philosophie, de la théologie ou de la morale. En fait, là où surgit l'originalité dans cette passion d'écrire, c'est du côté de la linguistique: française et huronne ${ }^{4}$.

Il faut souligner le souci constant de Potier d'enrichir le champ de l'enquête sur les manières de parler et les termes français. Depuis le temps des études en Belgique, tous les chemins parcourus seront des occasions d'enrichir la bibliothèque personnelle et le répertoire terminologique. Exilé à mille kilomètres de Québec, le voyageur dispose $\mathrm{d}$ 'une bibliothèque d'environ cent trente titres, $\mathrm{y}$ compris de nombreux dictionnaires (Trévoux, Joubert). Potier passe de la latinité de Térence, Cicéron, Horace, Ovide et Virgile au monde des lettres et transcrit 
quantité de chants (carmina), d'épitres, d'histoires poétiques, touche à la mythologie, constitue un répertoire des maximes, sentences, proverbes.

Et que dire du secteur des sciences, dont un grand nombre de cahiers nous manquent? Ici encore, une vaste érudition, pointilliste, comme s'il fallait conserver ce qui risque de disparaître ... à la frontière de l'inconnu!

Commencé dès les études en Belgique, le répertoire linguistique peut être considéré comme une frontière mouvante, dans la mesure où le chercheur avance d'étapes en étapes, saisit les expressions dites au fil des conversations, inscrit les noms de lieux, en particulier aux approches du Détroit.

Une fois rendu dans la région de Niagara, le père Potier constate que les voyageurs devront affronter le tumulte des vagues, bien avant d'atteindre l'île aux Bois-Blancs, sa destination. En quittant Niagara, c'est le terrible «roulin», i.e. le roulin «des vagues qui viennent se briser sur la grève». Ou alors, ce sont les «roulins fleuris, écumeux ... lame sourde», avec plus ou moins de «levée». . . ou «d'agitation des vagues»: «Si ce n'était la levée (ou agitation), nous filerions, irions bon train». Si les "petites lames» sont "courtes et sautillantes», il y a alors "clabotage» ou "clapotage» (comme on le dit encore). Et s'il fait «un vent impétueux», il en «cogne un bon coup». Quant au lac Érié, s'il est "faché, il lui faut 24 heures pour s'apaiser». S'il est «irrité ... . en colère ... enflé . . . le lac Ontario s'appaise d'abord».

On trouvera dans le lexique du parler français de Potier au Détroit des expressions encore en usage, telles que «un canot allège». Potier nous dit, par exemple que «Le frère Latour ne fait que bredasser", alors que nous disons plutôt «bardasser». Là où Potier écrit que cette «mauvaise horloge est une patracle», nous dirons que c'est une "patraque».

Voici d'autres expressions encore en usage aujourd'hui: «les poules cacassoient»; «renduire un mur»; «sa maison est sale comme une soue à cochon»; "après cette secousse». Nous disons souvent: «attends une secousse!». Potier note que «les Canadiens disent fisque ... et fisquer» pour «fixe et fixer». Évidemment, on dit encore, avec peu de variantes : «icit», «la boëte», «la quaisse [caisse] d'une horloge», «j'entendois les confessions d'arrache-pied», «débiter un poulet», «les petits hurons sont à jouailler», «Régis roupilloit». Potier note que Janis a dit: «Le frère Latour est tout dégommé», pour signifier qu'il est maladif. Dans le parler actuel, on dit de quelqu'un qu'il a été dégommé, s'il a perdu son emploi.

Quand Madame Daniau dit que «M. de Longueuil est un panier percé», elle veut dire qu'il «dépense tout son bien», un usage différent du nôtre, car nous dirions plutôt qu'il ne peut garder un secret. Madame Daniau disait aussi: «Ces enfans ne font que se tapoter, s'entre-frapper». Nous dirions qu'ils "passent leur temps à se chamailler». Si vous quittez vos amis, note Potier, vous avez droit au «coup de partance» ou «au coup 
de l'étrier». Avec un peu d'eau de vie ajouté au café, ce sera le «coup séraphique». Si vous conduisez votre voiture, évitez «le coup abénaquis» - grand coup d'une traite - qui pourrait vous «interboliser». Et alors! vous perdriez complètement la "tramontane». Ces quelques exemples suffiront de dégustation. Pour un vin plus raffiné, vous passez à la lecture de l'étude savante de Peter Halford.

Le père Potier ralentit le rythme de cette notation des manières de parler et des termes français vers 1750. Dans les trente années suivantes, d'autres priorités accaparent son temps. Il s'occupera davantage ailleurs, en divers domaines : en particulier de tout ce qui touche au «spectacle de la nature», titre d'un ouvrage de Pluche, qu'il note abondamment, sur tout le territoire des connaissances, fragmenté et entassé dans son encyclopédie personnelle. Une rédaction un peu sur tout, sans frontières et sans critique des contenus.

En somme, il a jeté l'ancre sur tout ce qui s'appelle "curiosité», au sens du dix-huitième siècle. Ce mot «tout» revient dans un petit essai rimé, symbole des totalités cueillies dans la lecture de l'événement, tant en Europe (document intitulé «le Tout de l'Europe»), qu'au Détroit (document intitulé «le Tout du Détroit»)(Écrits, doc. 17. VIII). Pour chaque pays d'Europe dont Potier décrit le caractère, il y a un portrait, en parallèle, des notables du Détroit, groupe social dont voici quelques noms: outre le Commandant, qui «se mèle de tout», il y a Berranger, De Muy, Godefroi, Navarre, Porlier, de Quindre, La Mothe, Demuisseau, Barrois, Cuillerier, Beaubien, Chicot, Réaume, Mme St-Martin, Jaqueau et, inévitablement, les Robes Noires, "accusées de tout». ${ }^{5}$

Il y a, en sourdine, un sens de l'humour, une place pour les jeux de l'esprit, comme dans cette histoire d'un procureur de collège, qui lança une assiette à la tête d'un régent, parce qu'il avait osé parler de la "matière subtile», de quoi jeter l'émoi dans la communauté des plus jeunes, que fascinait la philosophie de Descartes.

Dans cette mise en scène des "totalités", il y a encore beaucoup d'espace au-delà. Les marges, si étroites autour des écritures, sont, par leur silence, vastes comme les lacs, les forêts, les aurores boréales, les hivers et les territoires de chasse. La plume ne s'arrête que rarement, touche à tout, «les jésuites se trouvent partout», comme Potier lui-même, dans la mesure où son esprit s'aventure à tout capter. Qu'il s'agisse de Pierre Potier ou de toute autre mission que la sienne, il ne s'agissait pas de vaciller entre deux mondes, mais de «jeter l'ancre» dans un lieu à bâtir, en dépassant les frontières du monde ancien et les obstacles qui se dressent sur les routes de l'avenir.

En conclusion, disons que l'activité intellectuelle de Potier était pour ainsi dire sans frontières, sans limites! Son oeuvre témoigne une activité minutieuse, bien «ancrée» sur papier résistant, qu'une belle écriture, une 
encre de qualité — qualifiée de «bonne ancre [sic] ... melliflua» — a fixée une fois pour toutes, comme un sceau sur parchemin, ou mieux comme le métal sur le blason. Traduction d'une volonté qui dépasse tout l'horizon des lectures, ne s'arrête pas devant les obstacles. Pierre Potier, humaniste érudit, poursuit un projet longuement muri, élargissant l'horizon et la juridiction de la culture française, toujours à l'affut de livres pour alimenter sa bibliothèque. L'intimité de son décor devait contenir sur les rayons les fragments de sa quête encyclopédique.

Et nous voilà au paradoxe: d'une part, un esprit des plus ouverts, sur tout un monde de connaissances; d'autre part, un esprit des plus fermés, puisque Potier ne se pose guère de questions sur tout ce qu'il note. Cette curiosité envahissante saisit les manière de parler et les variantes du langage, sans pousser plus loin l'analyse linguistique. En somme, si l'on veut, une neutralité rassurante. Sur tout ce que la langue huronne peut exprimer, tant sur le plan linguistique que sur les modalités de sa pastorale catholique, rien. On ignore ce que les Hurons eux-mêmes en disaient. Territoire du silence des sources! Le «bonne ancre» de Potier seul demeure le leitmotiv, reliant le monde ancien au nouveau.

\section{ROBERT TOUPIN, S.J.}

Université Laurentienne de Sudbury

Institut Monserrat, St-Jérome

\section{Notes}

1 Voir Toupin, Les écrits de Pierre Potier (1996).

2 Ibid, doc. 2 (Journal de voyage) et 5-12 (Chemins).

3 Ibid, vol. II (Registres de catholicité).

4 Ibid, doc. 18 : "Façons de parler et terme français," avec notes historiques. Pour l'étude linguistique, consulter Halford. Pour les mss. sur la langue huronne, voir Écrits, p. 1164.

5 Les noms des familles françaises actuelles rappellent toujours le souvenir des pionniers de la culture: Drouillard, Janis, Baby, Bénéteau, Meloche, Touranjeau, Gervais, Dumouchelles, Beaubien, Cuillerier, Navarre, Porlier, Saint-Martin, Rhéaume, Chicot, Mailloux, Pajot, Monforton, Parent, l'Espérance, Bondy, Renaud, Pouget, Chaput, Rocheleau, Antaya, Pratt, Langlois, Labadie, Belleperche, Morand, Soullière, Chauvin, Pelletier, Maisonville, Goyau, Gignac, Girard, Rousseau. 


\section{Textes cités}

Halford, Peter W. Le français des Canadiens à la veille de la conquête. Témoignage du père Pierre Philippe Potier, s.j. Ottawa: Presses de l'Université d'Ottawa, 1994.

Toupin, Robert. «L'érudition et la fonction du savoir au XVIII ${ }^{\mathrm{e}}$ siècle : Pierre Potier chez les Hurons du Détroit». Man and Nature / L'homme et la nature. London, 1982. 165174 .

"Les coordonnées d'un nouvel espace dans les écrits de Pierre Potier, jésuite belge chez les Hurons du Détroit». Revue de l'Université d'Ottawa 56.1 (janv-mars 1986): 67-76. No spécial sur le thème de l'Ailleurs.

. «Les routes de canot et de terre au pays des Illinois et dans la vallée de la Ouabache au XVIII ${ }^{\mathrm{e}}$ siècle: les recueils de Pierre Potier». Actes du Premier Congrès international sur la toponymie française de l'Amérique du Nord. Québec, 1986. 446-454.

"Testaments et recommandation de l'âme chez les Hurons du Détroit au XVIII siècle». Archivum Historicum Societatis Iesu. Rome, 1988. 180-185.

. Arpents de neige et Robes Noires. Brève relation sur le passage des Jésuites en NouvelleFrance. Montréal: Bellarmin, 1991.

. Les écrits de Pierre Potier. Ottawa: Presses de l'Université d'Ottawa, 1996. Vol. I : La culture savante en Nouvelle-France au XVIII siècle. Vol. II: Registres de catholicité. Et bibliothèque du père Potier. 